\title{
Magnetic properties of CHAMP and their effects on in-orbit calibration
}

\author{
H. Lühr ${ }^{1}$, F. Yin ${ }^{2}$, and R. Bock ${ }^{3}$ \\ ${ }^{1}$ Deutsches GeoForschungsZentrum, GFZ, 14473 Potsdam, Germany \\ ${ }^{2}$ Dept. Space Physics, Wuhan University, 430079 Wuhan, China \\ ${ }^{3}$ European Space Agency, ESTEC, 2201 AZ Noordwijk, The Netherlands \\ Correspondence to: H. Lühr (hluehr@gfz-potsdam.de)
}

Received: 8 November 2012 - Revised: 4 February 2013 - Accepted: 13 February 2013 - Published: 4 March 2013

\begin{abstract}
CHAMP has so far been the most successful magnetic field mission. For achieving that, special effort had to be invested in building a magnetically clean spacecraft. The magnetic moment of the spacecraft is about $1 \mathrm{Am}^{2}$. In this article we introduce a new method that allows the determination of the magnetic moment during the mission from the average current strength of the magneto-torquers. In order to achieve precise field vector data, the readings of the fluxgate magnetometer are routinely calibrated against the absolute Overhauser measurements. A reanalysis of all the magnetic field data is performed which takes also into account small disturbances from the power system. Uncertainties of the final magnetic field data are estimated to be of the order of $0.1 \mathrm{nT}$.
\end{abstract}

\section{Introduction}

The German satellite mission CHAMP (CHAllenging Minisatellite Payloads) was dedicated to systematic surveys of Earth's gravity and magnetic fields, as well as to performing atmospheric sounding (see Fig. 1). The spacecraft was launched on 15 July 2000 into a circular, near-polar orbit at $456 \mathrm{~km}$ altitude during the solar maximum phase, and it reentered the atmosphere on 19 September 2010 after having passed the deep and extended solar minimum period. More details of the mission and initial results can be found in Reigber et al. $(2003,2005)$. The continuous recordings over $10 \mathrm{yr}$ provide an excellent dataset for detailed modelling of the Earth's potential fields, but it also provides the opportunity to deduce the magnetic properties of the spacecraft and the characteristics of the sensors.

Investigating the geomagnetic field was one of CHAMP's prime science objectives. For that purpose the satellite carried a fluxgate vector magnetometer (FGM) and an Overhauser scalar magnetometer (OVM). Both are accommodated on a 4-m-long boom.

A prerequisite for a magnetic field mapping mission is a magnetically clean spacecraft. All instruments had to pass magnetic tests before installation. To each of them an accep- tance level was assigned. In addition, a magnetic system test was performed before launch. The purpose of this test was to obtain the magnetic properties of the system and to provide numbers for certain parameters which are needed for the processing of the magnetic field measurements. Among them are the magnetic moment of the spacecraft, its static induced field, stray fields from electric currents, signatures of the magneto-torquers, and the crosstalk from the fluxgate onto the Overhauser magnetometer.

During the mission the fluxgate magnetometer was routinely calibrated against the absolute Overhauser magnetometer. As a result of that procedure, the 9 fluxgate parameters $(3$ scale factors, 3 offsets and 3 sensor misalignment angles) are determined. Looking at the temporal evolution of these parameters over $10 \mathrm{yr}$ gives interesting insights into the dependencies of the sensors on the various ambient influences.

An underlying assumption of the scalar calibration approach is that all the differences between the fluxgate and the Overhauser readings can be attributed to imperfections of the 9 fluxgate parameters. Any contribution of spacecraft systems to this difference may falsely be absorbed by some of these parameters. For the reanalysis of the whole CHAMP 
Table 1. Magnetic field disturbances caused by the CHAMP spacecraft at the location of OVM, as derived from the system magnetic test.

\begin{tabular}{lrrr}
\hline Field components & X (nT) & Y (nT) & Z (nT) \\
\hline Remanent field & $<0.1$ & -0.35 & 0.2 \\
Static induced field @ 30 $\mu \mathrm{T}$ & 0.45 & -0.18 & -0.15 \\
FGM-to-OVM crosstalk @ 30 $\mu \mathrm{T}$ & -0.63 & 0.4 & 0.45 \\
\hline
\end{tabular}

magnetic field dataset we have extended our error model and allowed for additional error sources related to the power system.

In the sections to follow, we outline shortly the magnetic cleanliness programme for CHAMP and then describe the structure of the routine data processing. In Sect. 4 the results of scalar calibration are presented, and in Sect. 5 an extended error model is introduced. Section 6 summarizes the main results and makes suggestions for future magnetic field missions like ESA's Swarm project.

\section{Magnetic cleanliness programme}

For CHAMP, as a low-cost project, we had to avoid demanding magnetic cleanliness programmes. The approach selected was a magnetic screening of all instruments before installation. Each unit was taken through a three-step procedure. Measurements of magnetic moment were performed in the following states: "as received", after magnetization in a $240 \mathrm{~A} \mathrm{~m}^{-1}$ (3 Gauss) field, and after demagnetization. For acceptance the last result was considered. The two other values were just noted for characterization. The general acceptance level was $10 \mathrm{~mA} \mathrm{~m}^{2}$ per $\mathrm{kg}$ of mass for any of the units. Instruments which did not stay below this value needed special considerations. For boom instrumentation, e.g. the two star camera heads, of course more stringent rules were applied. More than $90 \%$ of the units passed their magnetic test without extra effort.

Shortly before the launch a system magnetic test was performed in the magnetic test facility (MFSA) of the IABG (see table before the references for further acronyms). During this test it was discovered that the two cold gas pressure tanks, which had not been screened magnetically, exceeded by far the requirement. Their magnetic moments were of the order of $1.5 \mathrm{Am}^{2}$ each. Luckily, the tanks were installed in antiparallel directions such that the magnetic moments cancelled each other to a large extent. The total magnetic moment of the spacecraft amounted to $1.1 \mathrm{Am}^{2}$. From the disturbance dipoles determined experimentally during the test, we calculated residual fields of less than $0.1 \mathrm{nT}$ in along-track direction, $-0.35 \mathrm{nT}$ in cross-track and $0.2 \mathrm{nT}$ along nadir at the position of the OVM.

The static induced field, caused by soft magnetic material, was also determined. In an ambient field of $30000 \mathrm{nT}$ it causes an enhancement in along-track direction of $0.45 \mathrm{nT}$;
Table 2. Maximum magnetic field disturbances caused by the three torquer coils at the position of OVM. The torquer correction matrix was determined during system magnetic test and validated in orbit.

\begin{tabular}{lrrr}
\hline Field components & $\mathrm{X}(\mathrm{nT})$ & $\mathrm{Y}(\mathrm{nT})$ & $\mathrm{Z}(\mathrm{nT})$ \\
\hline Torquer coil 1 & 2.2 & -0.8 & -0.8 \\
Torquer coil 2 & 0 & -3.0 & 0 \\
Torquer coil 3 & -0.18 & 0.15 & -3.5 \\
\hline
\end{tabular}

in cross-track and nadir direction it caused field reductions by $0.18 \mathrm{nT}$ and $0.15 \mathrm{nT}$, respectively. This effect is partly compensated by the crosstalk of the FGM onto the OVM measurements. For the crosstalk we found under above mentioned ambient conditions a reduction of $0.63 \mathrm{nT}$ in alongtrack, enhancements of $0.4 \mathrm{nT}$ in cross-track and 0.45 in nadir directions. Although all these effects add up to less than $1 \mathrm{nT}$ at OVM, their influence has been taken care of in the data processing. The above-mentioned magnetic features of CHAMP are summarized in Table 1. Here and elsewhere the components are defined as the following: $x$-axis points in flight direction, $\mathrm{z}$ towards nadir, and the $\mathrm{y}$-axis completes the triad pointing in cross-track direction.

The CHAMP satellite was equipped with magnetotorquers for supporting the attitude control of the spacecraft. Magnetic disturbances caused by the three air coils had to be determined precisely in order to allow for an efficient correction of the measurements during the mission. In Table 2 the maximum field values are listed that are generated by the three torquers (@300mA) at the position of OVM. More details of the CHAMP magnetic system test can be found in Lühr et al. (2000).

\section{Routine data processing}

A basic assumption of the CHAMP data processing approach is that the measurements of the Overhauser magnetometer are absolutely correct. Therefore, this instrument is taken as reference for the mission. It is thus important to remove all additional fields generated by the spacecraft properly from the OVM readings in order to obtain the correct ambient field strength. The major processing steps are listed in Fig. 2.

Step 1 takes care of any delay between the time stamp and actual epoch of the measurement. The internal oscillator of the OVM is checked every minute against the frequency standard of the GPS system. Deviations are considered in step 2. At step 3 the remanent spacecraft field and magneto-torquer fields are corrected; in step 4 the static induced field and the crosstalk from FGM are taken care of. After these manipulations the OVM readings properly represent the ambient field strength.

We have invented a new approach for tracking secular changes of the spacecraft remanent magnetic field during the mission. It relies on the assumption that the field produced by 


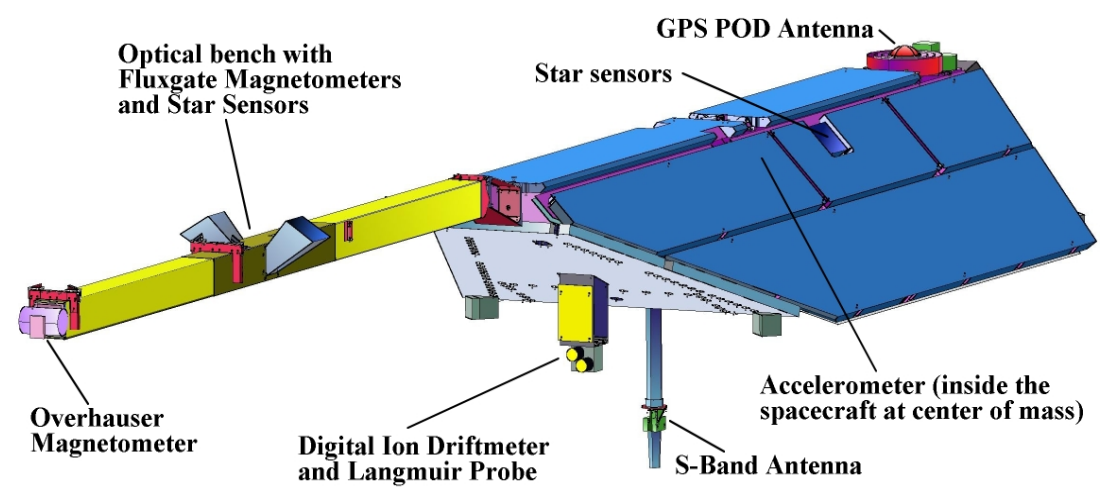

Figure 1. Schematic drawing of the CHAMP satellite with all payload instruments assigned. CHAMP flew with the boom in forward direction.

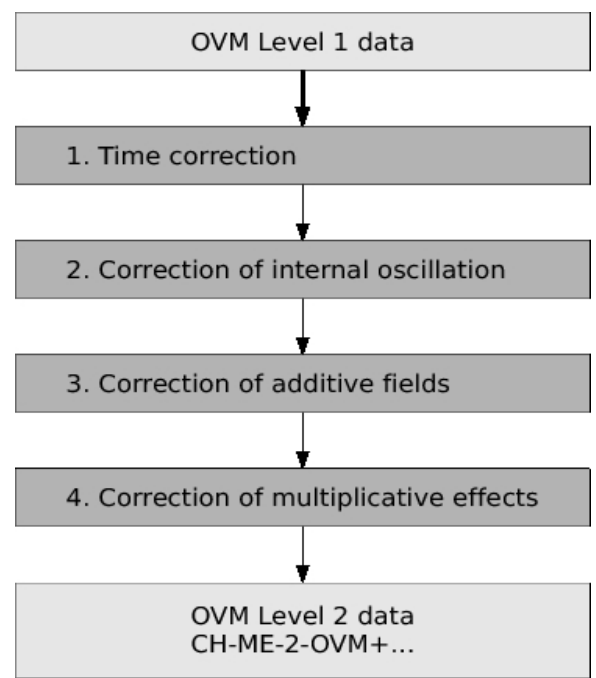

Figure 2. Major processing steps of OVM data.

the magneto-torquers on average just compensate the magnetic moment of the spacecraft. All control activities cancel out over sufficiently long periods. In the case of the very symmetric shape of the CHAMP spacecraft this seems to be reasonable. The magneto-torquers on CHAMP operated continuously and were practically able to maintain the full attitude control. During normal operation only some 5 thruster firings per orbit were typically needed for supporting the attitude control when limits are exceeded.

Figure 3 shows the temporal variation of the torquer current readings. The currents through all the three coils vary primarily with the local time of the orbital plane. By taking running averages over 260 days we suppressed the oscillations. Within 260 days the CHAMP orbital plane precessed through all local times. The average curves (black crosses) are considered to represent the inverse of the spacecraft magnetic moment. Each torquer coil generated a magnetic moment of $20 \mathrm{Am}^{2}$ per ampere. The right-side scale of Fig. 3 re-

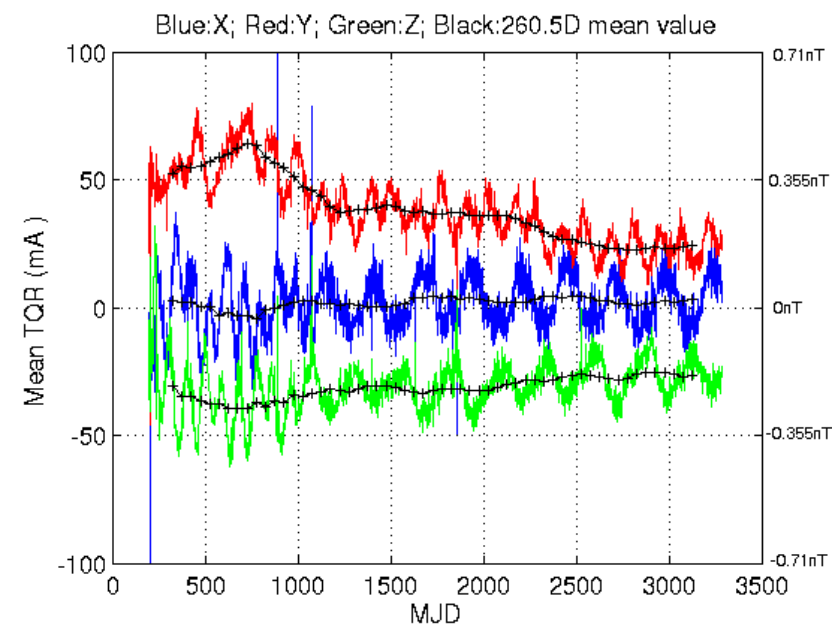

Figure 3. Variations of the magneto-torquer currents. The coloured lines represent daily averages; black crosses are running averages over 260 days. The right-side scale reflects the equivalent magnetic field at OVM. MJD: Modified Julian Day.

flects the magnetic field that a dipole at the spacecraft centre with corresponding moment would generate at the position of OVM. For validating our assumption we may go back to the test results from the MFSA. There we obtained a spacecraft field of $X<0.1 \mathrm{nT}, Y=-0.35 \mathrm{nT}$ and $Z=0.2 \mathrm{nT}$ at the position of OVM (Table 1). These numbers fit very well the initial field values of the three components in Fig. 3, but have opposite signs, as expected. The average curves (black crosses) are used for removing the remanent field effect from the OVM measurements. We find an interesting temporal evolution in particular of the y-axis. After an initial increase there are two clear decreases of the y-axis spacecraft field. Periods of decrease correlate well with the times of the three orbit rise manoeuvres on MJDs 891, 1073 and 2277. During those events significant amounts of cold gas were used. Obviously, the magnetization of the cold gas tanks, main contributors to the spacecraft moment, became smaller with the decreasing 


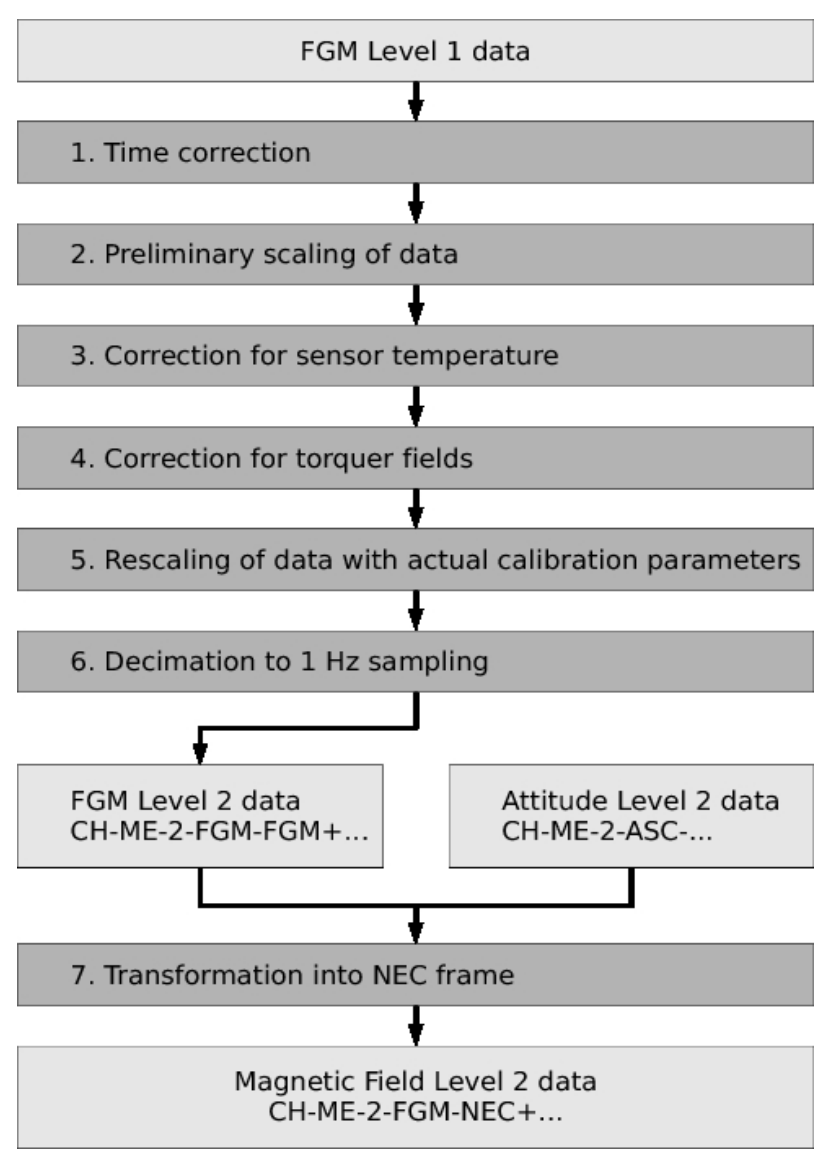

Figure 4. Major processing steps of FGM data.

pressure. The gradual decrease of magnetization between the manoeuvres can be related to the continuous consumption of gas for attitude control. With the help of this approach, we could properly track the temporal evolution of the spacecraft field throughout the 10-yr mission. This method may well be applicable also for other missions like ESA's Swarm.

The processing of FGM data, as shown in Fig. 4, relies heavily on the precision of OVM data. Only effects varying on scales shorter than a day are corrected for here, e.g. torquer fields. Slowly changing effects like the spacecraft field are taken care of by the scalar calibration of the FGM data against the OVM readings. The FGM data processing follows a similar sequence as that for OVM. Output data from Step 4 are used for the scalar calibration. In Step 5 the newly obtained parameters are applied, and fully calibrated vector data are generated.

Since the scalar calibration is such an important tool for the CHAMP mission, it is shortly introduced here. The aim is to obtain identical scalar field values from OVM and FGM readings. In order to achieve that, the vector measurements are expanded by possible error contributions.

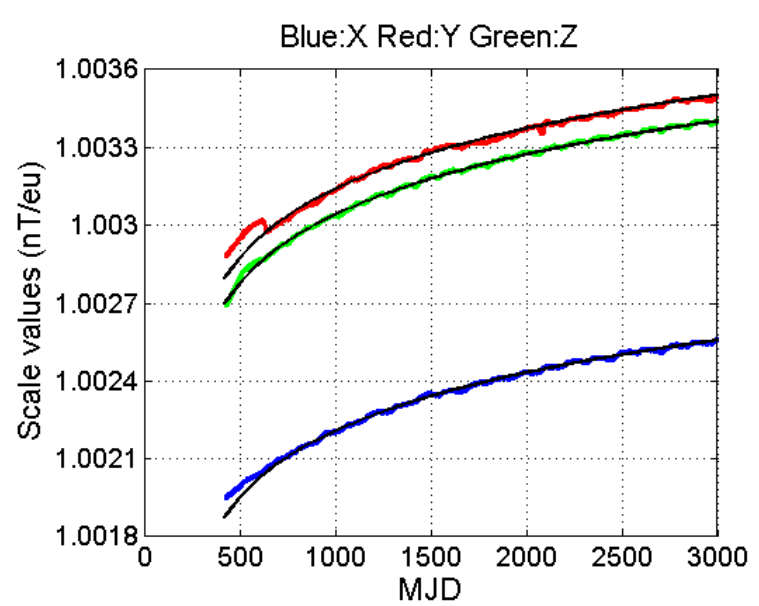

Figure 5. Temporal evolution of the FGM scale factors.

$$
\begin{aligned}
B_{\mathrm{OVM}}^{2} & =\left(S_{x} E_{x}+O_{x}+E_{y} \cos (X, Y)+E_{z} \cos (X, Z)\right)^{2} \\
& +\left(S_{y} E_{y}+O_{y}+E_{z} \cos (Y, Z)\right)^{2}+\left(S_{z} E_{z}+O_{z}\right)^{2},
\end{aligned}
$$

where $S_{X}, S_{Y}, S_{Z}$ are the scale factors, $O_{X}, O_{Y}, O_{Z}$ are the offset values, $(X, Y)(X, Z)(Y, Z)$ are the angles between sensor axes and $E_{X}, E_{Y}, E_{Z}$ are the readings of the FGM. By considering measurements from a full day, the 9 FGM parameters can be determined that provide the best fit between FGM and OVM data. During the mission the scalar calibration has routinely been applied. More details of the CHAMP magnetic field data processing can be found in Mandea et al. (2010).

\section{Temporal evolution of FGM}

The long and continuous CHAMP dataset provides the opportunity to investigate the long-term characteristics of the FGM instrument. Here we present the evolution of the 9 fluxgate parameters. Results may give hints for further improvements of the instrument, but are also of interest for reprocessing of the data.

Starting with the scale factors, as shown in Fig. 5, we see a continuous increase. The sensitivity becomes larger by less than $0.1 \%$ over the course of $10 \mathrm{yr}$. All three components follow the same trend. Determined variations can be approximated very well by similar logarithmic functions (black lines). This suggests an annealing of some electronic components (semi-conductor) as the cause of the change. According to the instrument provider, the drift is caused by an aging of the reference voltage source, commonly used for the three ADCs, one for each component (J. M. G. Merayo, personal communication, DTU, 2010).

There is a jump in the y-component scale factor early in the mission. This is in response of a calibration manoeuvre where the spacecraft had been flown for $12 \mathrm{~h}$ with an attitude 


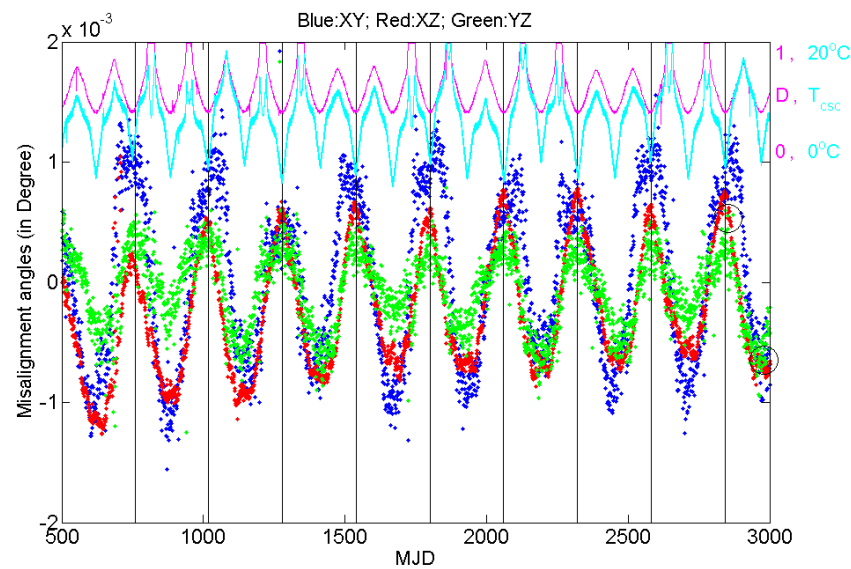

Figure 6. Temporal variation of the angles between the sensor axes. Vertical lines mark times of 12:00 orbital local time. The cyan curve shows the sensor temperature and the magenta curve represents the fraction of an orbit that is in sunlight.

rotated by $90^{\circ}$ about the vertical axis. In this configuration the y-axis points in flight direction and the scale factor, $S_{y}$, can be determined reliably. During the days before the manoeuvre this parameter was obviously biased. It is known that the scale factor of the y-axis is ill determined by the scalar calibration due to the small geomagnetic field strength in east-west direction.

Very favourable results are obtained for the angles between the sensor axes. As can be seen in Fig. 6, the angles do not show any long-term trend exceeding 1 arcsec over the course of the mission. Constant values have been subtracted before plotting in order to expand the scale.

There is an apparent variation of all three angles by about $0.001^{\circ}( \pm 3 \mathrm{arcsec})$ at a period of 260 days. Maxima coincide with an ascending node of the orbit at 12 o'clock. Since the sensor temperature changes at double the frequency as the angles, it is difficult to find a physically plausible explanation for the apparent variations. We will revisit this issue in the next section.

The third quantity derived from scalar calibration is the magnetometer offset. As shown in Fig. 7, there is hardly any long-term trend and the deflections do not exceed $0.5 \mathrm{nT}$ too often. Here again we find dominant variations at 130 and 260-day periods.

In summary, there is a significant change of sensitivity with time, but it can be approximated by a simple function. The other 6 parameters can be considered constant over the whole mission.

\section{Extended error model}

By assessing the temporal evolution of the FGM parameters we got insight in the instrument characteristics. It seems possible to use analytical expressions for the 9 parameters that

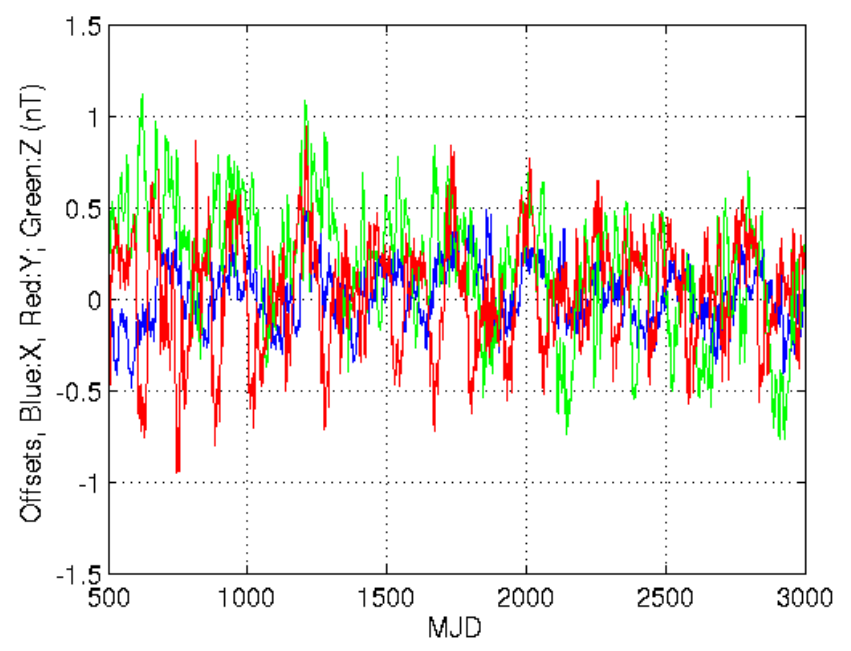

Figure 7. Temporal evolution of the FGM offsets. Constant values have been subtracted.

describe sufficiently well their variations over the full 10-yr period. A suitable function for the scale factors (also used in Fig. 5) is

$S=S_{0}+S_{1} \ln (\mathrm{MJD}-150)$,

where $S_{0}$ and $S_{1}$ are parameters to be determined for each component, MJD is the modified Julian day. For the offsets and sensor angles we choose constant values, the mean values over the mission.

When the scaling properties of the FGM measurements are known, it is possible to extend the error model of CHAMP magnetic field data. Yin and Lühr (2011) have presented an approach for interpreting the remaining residuals, $\Delta B$, between FGM and OVM readings. They introduce a model that solves for additional error contributions

$$
\begin{aligned}
\Delta B & =\left|B_{\mathrm{FGM}}\right|+\left\{f_{x}\left(p_{1}\right) \frac{B_{x}}{|B|}+f_{y}\left(p_{1}\right) \frac{B_{y}}{|B|}+f_{z}\left(p_{1}\right) \frac{B_{z}}{|B|}\right. \\
& \left.+g_{x}\left(p_{2}\right) \frac{B_{x}}{|B|}+g_{y}\left(p_{2}\right) \frac{B_{y}}{|B|}+g_{z}\left(p_{2}\right) \frac{B_{z}}{|B|}+\ldots\right\}-B_{\mathrm{OVM}},
\end{aligned}
$$

where $p$ are the parameters that control the disturbances and $f, g$ are functions that describe the relation between parameter and disturbance.

Here we are presenting two examples of magnetic signatures which are caused by the power supply system. When the satellite comes from the nightside into daylight, large currents are flowing in the solar cells for charging the battery. When the battery is fully charged, the current strength drops suddenly from more than $10 \mathrm{~A}$ to about $3.5 \mathrm{~A}$ - the operational level of power consumption (Fig. 8, bottom).

As can be seen in Fig. 8 (top), there is a small response in $\Delta B$ (the difference between FGM and OVM readings) when the current drops. The ratio of step sizes between these two sudden changes is used to quantify the magnetic effect of 

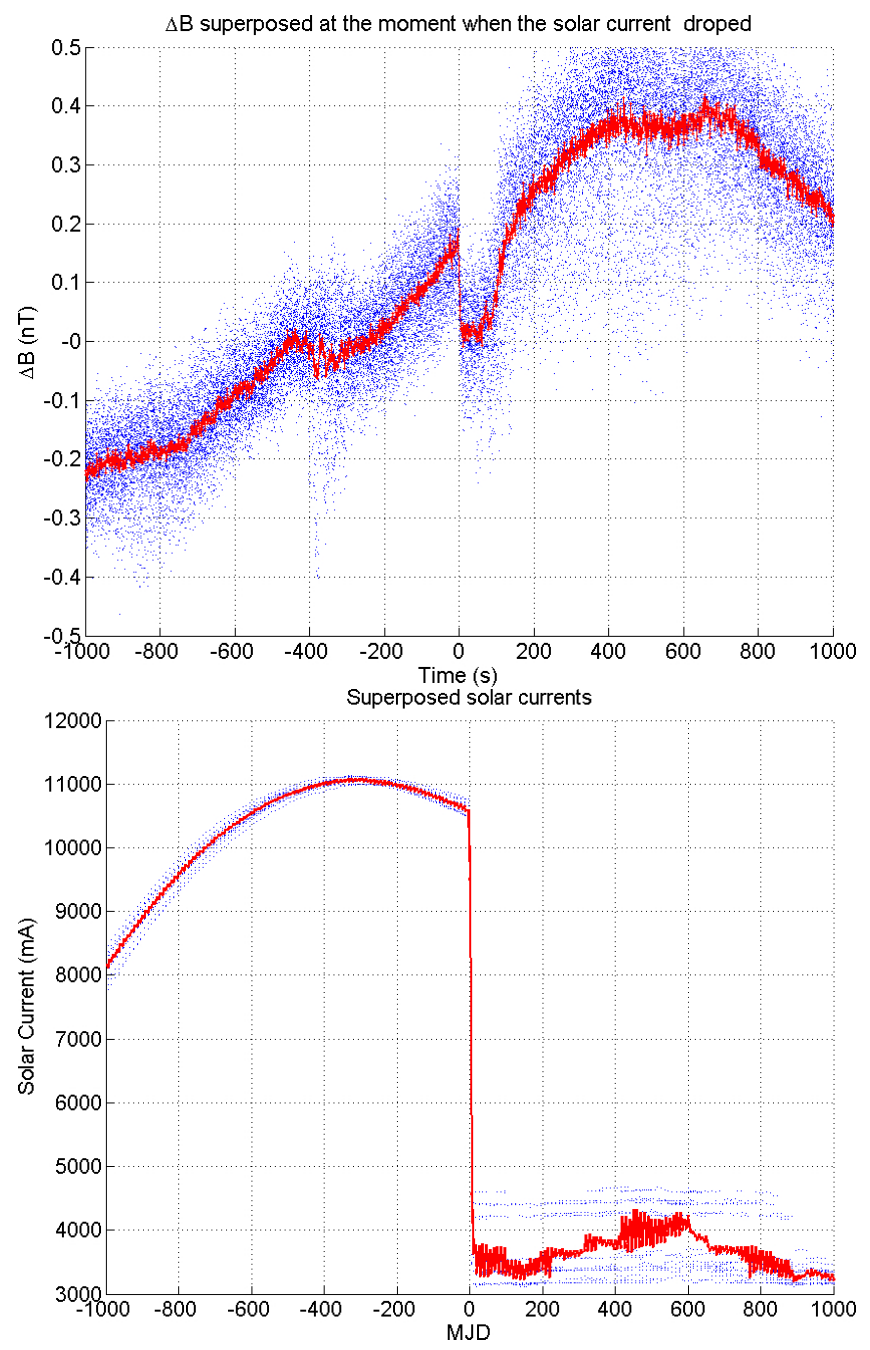

Figure 8. Magnetic signature of solar array current. The red lines mark averages of the individual readings (blue dots). In sunlight the battery is charged. When it is full, the current strength drops abruptly. The related magnetic signature is of the order of $0.15 \mathrm{nT}$ at the OVM.

solar array currents in general. CHAMP has solar panels on both sides (see Fig. 1). It is expected that the magnetic effects are different from the two panels. For that reason the size and the orientation of the magnetic disturbance vector will be orbit local time dependent.

We have applied Eq. (3) for interpreting the magnetic signature of Fig. 8. As controlling parameter we used the measured total solar array current. The equation was solved for the functions $f_{x}$ and $f_{z}$. Solutions for $f_{y}$ were not stable because of the weak ambient field in east-west direction. Figure 9 shows the temporal variation of magnetic disturbance functions for the $\mathrm{x}$ - and $\mathrm{z}$-components. In order to support the interpretation vertical magenta lines mark the times of 12-h orbits and cyan lines the $24-\mathrm{h}$ orbits. The disturbances show clear orbit local time dependence. There is a reversal of the scaling factor between hours around 06:00 and 18:00 LT.

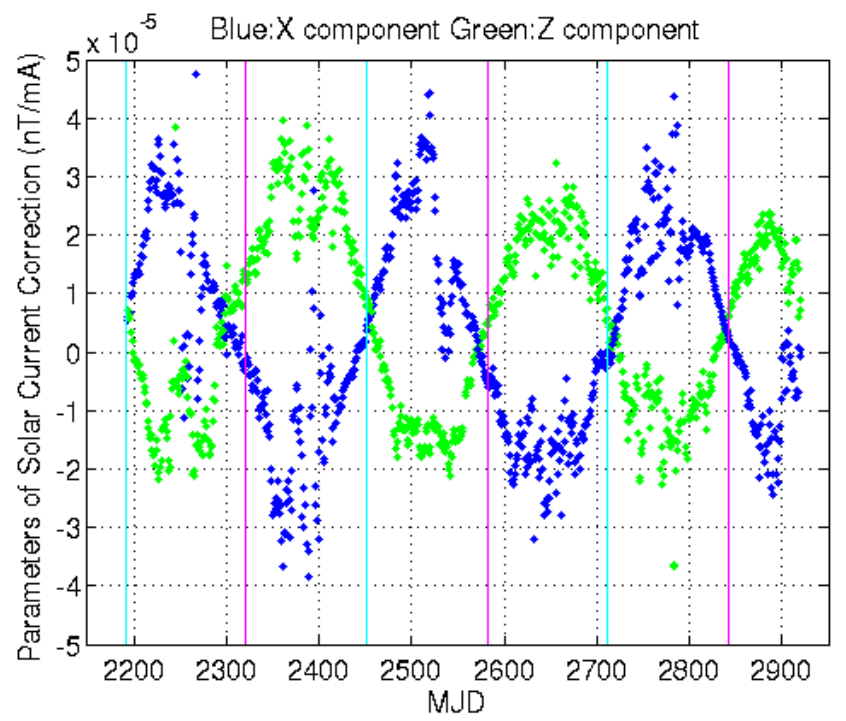

Figure 9. Ratio between solar current strength and magnetic effects in the $\mathrm{x}$ - and $\mathrm{z}$-components. Vertical magenta lines mark orbits at 12:00 and cyan at 24:00 LT.

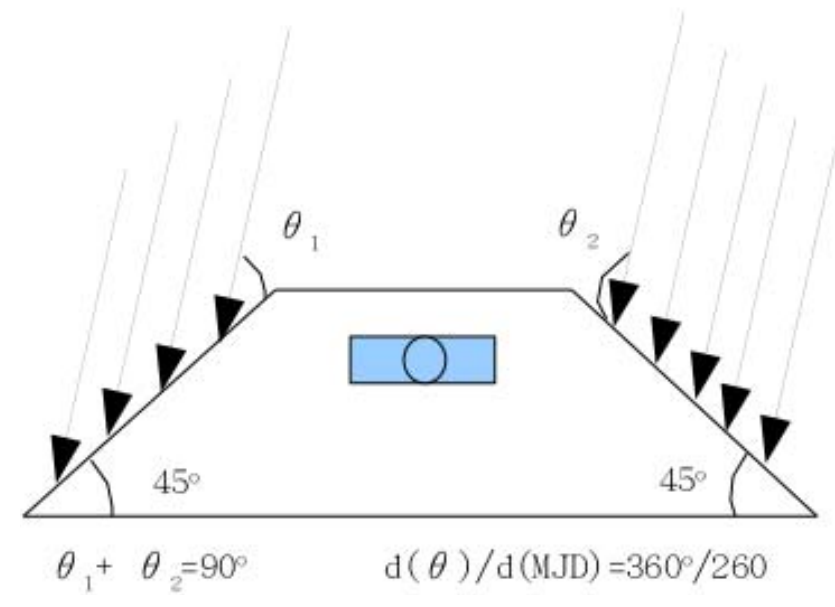

Figure 10. Illumination of the solar panels on the two sides during an orbit close to noon.

For the interpretation of the disturbance function we have to consider the illumination conditions of the solar arrays on the two sides of the satellite (see Fig. 10). On orbits near dawn and dusk the sun is illuminating only one panel. Thus, at times halfway between the vertical lines in Fig. 9 magnetic disturbances come only from one panel. Effects of the left and right side follow each other after 130 days. It can be seen that the two solar panels cause magnetic disturbances with opposite signs. The situation where only one side of the satellite is illuminated is valid for orbits in the local time sectors 03:00-09:00 and 15:00-21:00. Therefore, the functions in Fig. 9 are flat at tops and bottom over these sectors.

The two solar panels generate approximately the same disturbances per ampere but with opposite signs. When 
CHAMP passed the equator close to noon, both panels were illuminated and the disturbances almost cancelled. For estimating the magnetic effect, we have to multiply the disturbance function with the solar current value. For orbits around dawn and dusk, when the scaling factors in Fig. 9 are highest, no battery charging is required. Thus, expected disturbances are below $0.1 \mathrm{nT}$. Similar values are obtained for noon orbits. Even though currents go up to $16 \mathrm{~A}$, the effects from the two panels almost cancel. Largest magnetic signatures come from 03:00, 09:00, 15:00 and 21:00 LT orbits when only one panel is active, but already sizeable charge currents are needed (see example in Fig. 8).

For a proper characterization of the effect, a disturbance function for each of the two panels is required. Unfortunately, CHAMP does not provide individual readings of the currents from the two solar panels, but only the total solar current is monitored. If these two housekeeping values were available, they could directly serve as controlling parameters for correcting the magnetic disturbances. In order to mitigate this problem, we have modelled the illumination conditions of the two solar panels for all orbits and estimated the individual current values under the condition that the sum of both is known. Based on these current estimates the magnetic field readings were corrected.

In Sect. 4 we had noted that the angles between sensor axes showed some apparent oscillations. In the reanalysis of the CHAMP magnetic field data, we have used constant values for the angles. As a result the residuals, $\Delta B$, between FGM and OVM data showed some systematic variations. For the interpretation of the residuals we again employed Eq. (3). We found that the battery discharge current was a suitable controlling parameter, $p$. The task is here again to determine the function $g$ for each component that describes the relation between battery current and disturbance.

Figure 11 as a composite figure shows in black the battery discharge current. In the middle part the spacecraft is in sunlight, thus no current comes from the battery. The broad blue curve is a synchronous stack of all $\Delta B$ curves of one day, and the red in the middle represents the mean values. This mean curve can well be reproduced by Eq. (3) (see overlaid green curve) when suitable functions for $g_{x}$ and $g_{z}$ are used. The two functions, $g_{x}$ : magenta and $g_{z}$ : cyan, vary in phase with the battery current.

As can be seen in Fig. 11, the correlation between the residuals (blue curve) and the battery current is not obvious at first sight. In case of an ascending orbit (top panel) the residuals show a plus/minus oscillation around the equator crossing, while for descending orbits $\Delta B$ exhibits a minus/plus bipolar signature. Only by decomposing $\Delta B$ into the disturbance functions according to Eq. (3) can we obtain the in-phase relations with the battery discharge current. The disturbances have amplitudes of about $0.5 \mathrm{nT}$ in the zaxis and half of it in the $\mathrm{x}$-axis at FGM location. Here again the $y$-axis contribution cannot be resolved. These disturbances are most pronounced on noon/midnight orbits, when
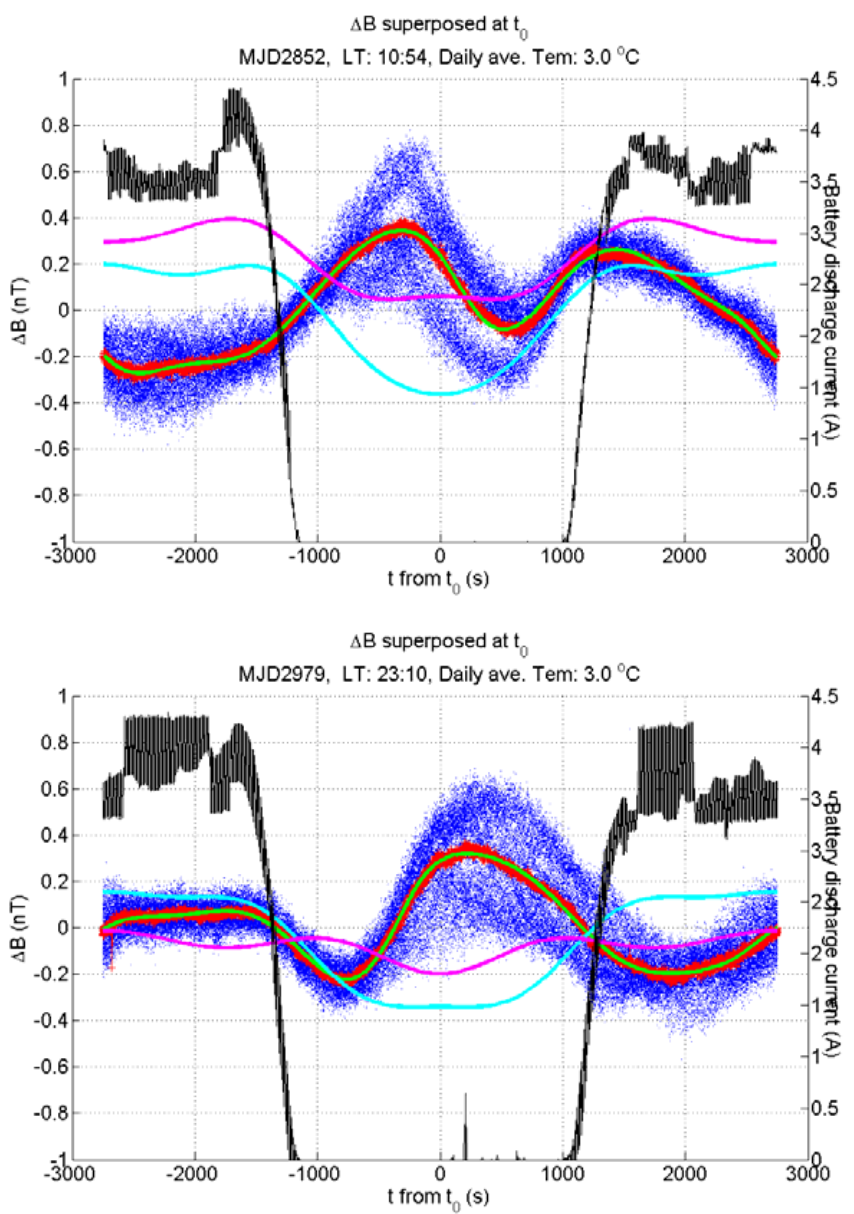

Figure 11. Magnetic signature of battery discharge current. During darkness the current (black) is high and it goes to zero in sunlight. Quite different $\Delta B$ curves (blue dots and red average curve) appear around noon for ascending (top) and descending (bottom) orbits. By modelling the $\Delta B$ effect (green curve), the magnetic disturbances of the $\mathrm{x}$-axis (magenta) and $\mathrm{z}$-axis (cyan) are estimated.

eclipses last longest and vanish during dawn/dusk full-sun orbits. In Sect. 4 we had shown that the disturbances were misinterpreted by the scalar calibration as variation of intersensor axis angles. As expected, the largest apparent deflections were found on noon/midnight orbits and no effect on dawn/dusk orbits.

The only other source of disturbance that was discovered is the nearby start tracker. Whenever one of the cameras went into saturation, e.g. by grazing incidence of sunlight a transient signature of some $0.2 \mathrm{nT}$ was recorded by the FGM. Conversely, during full saturation there was no effect. Due to a missing parameter characterizing this disturbance we could not remove it.

From the results shown in this section it can be concluded that dynamic disturbances, such as the magnetic effects coming from the power supply system, have to be corrected before the scalar calibration is applied. 


\section{Summary}

In this article we have given an impression of the magnetic properties of the CHAMP satellite. As a mission serving simultaneously gravity and magnetic field research, certain compromises had to be made, e.g. a limited boom length. Nevertheless, the magnetic field mission can be regarded as very successful. Here we are summarizing the important magnetic features of the spacecraft and the relevant instruments.

1. The spacecraft was designed and built obeying the rules of magnetic cleanliness. This includes the avoidance of magnetic materials, use of twisted cables for power supplies, avoiding ground loops in the electrical architecture etc. These measures helped to keep the magnetic moment of the spacecraft at a level of about $1 \mathrm{Am}^{2}$. The accommodation of the magnetometers on a 4-m boom helped in that remaining magnetic signatures of the spacecraft caused only small disturbances.

2. A new approach is presented here for monitoring the spacecraft remanent magnetic field during the mission. By assuming that the magneto-torquer action on average just cancels the magnetic moment of the satellite, the applied currents can be used to estimate the magnetic evolution of the spacecraft. In case of CHAMP this method provided reliable results, which were verified in the commissioning phase by comparison with prelaunch calibration data. During the mission the magnetization changed approximately in line with the cold gas tank pressure. Confirming the dominance of the tanks for the CHAMP magnetic properties.

3. For maintaining a high reliability of the CHAMP magnetic field data during the mission, the absolute scalar Overhauser magnetometer was used for calibrating routinely the readings of the fluxgate vector magnetometer. This so-called scalar calibration revealed that the sensitivity of the FGM continuously increased by less than $0.1 \%$ in $10 \mathrm{yr}$. The increase follows a logarithmic function in time. Conversely, the instrument offsets and the angles between the sensors showed no long-term trend.

4. In a reanalysis of the CHAMP magnetic field data after completion of the mission the characteristics of the FGM were described by analytical functions: logarithmic functions for the scale factors and constant values for offsets and angles. The remaining residuals between FGM and OVM readings (of order $0.5 \mathrm{nT}$ ) were then interpreted as disturbances from the spacecraft. Convincing models could be constructed that relate the residuals to solar array currents and battery discharge currents. When these contributions are removed the residuals stay in the range of $0.1 \mathrm{nT}$.
5. We have shown that the scalar calibration is a useful tool for characterizing the temporal variation of the FGM properties. But in case of disturbances varying with the orbital period or harmonics of it misinterpretations will result. It is thus important that influences coming e.g. from temperature or power supply are corrected before the scalar calibration is applied. An important prerequisite for that is the availability of housekeeping data, such as temperature and currents, which are needed for parameterizing the disturbances.

The results presented here may be useful for other magnetic field mapping missions. In particular the Swarm satellites are quite similar to CHAMP. The lessons learned may be considered for the evaluation of the Swarm data. Most of the magnetic disturbances discussed here had amplitudes of less than $1 \mathrm{nT}$, even though it is desirable to reduce them further since they are of systematic character. Even in the case of long data series they will not disappear, but will produce systematic errors in the derived field models on various temporal and spatial scale lengths.

\section{Abbreviation and acronyms}

$\begin{array}{ll}\text { CHAMP } & \text { Challenging Mini-Satellite Payload } \\ \text { CoG } & \text { Centre of Gravity } \\ \text { ESA } & \text { European Space Agency } \\ \text { FGM } & \text { Fluxgate Magnetometer } \\ \text { GPS } & \text { Global Satellite System } \\ \text { IABG } & \text { Industrie-Anlagen-Betriebs-Gesellschaft } \\ \text { MFSA } & \text { Magnet-Feld-Simulations-Anlage } \\ \text { MJD } & \text { Modified Julian Day } \\ \text { NEC } & \text { North-East-Centre coordinate frame } \\ \text { OVM } & \text { Overhauser Magnetometer }\end{array}$

Acknowledgements. The authors thank M. Rother, J. Rauberg and I. Michaelis for their dedicated effort in processing the CHAMP magnetic field data. The CHAMP mission was sponsored by the Space Agency of the German Aerospace Center (DLR) through funds of the Federal Ministry of Economics and Technology, following a decision of the German Federal Parliament (grant code 50EE0944).

Edited by: M. J. da Silva

Reviewed by: two anonymous referees

\section{References}

Lühr, H., Bock, R., and Rother, M.: CHAMP Magnetic Test Report, CH-GFZ-TR-2000, GFZ Technical Report, 2000.

Mandea, M., Holschneider, M., Lesur, V., and Lühr, H.: The Earth's magnetic field at the CHAMP satellite epoch, in: System Earth via Geodetic-Geophysical Space Techniques, edited by: Flechtner, F., Gruber, T., Güntner, A., Mandea, M., Rothacher, M., 
Schöne, T., and Wickert, J., Advanced Technologies in Earth Sciences, 475-526, doi:10.1007/978-3-642-10228-8_42, SpringerVerlag Berlin-Heidelberg, 2010.

Reigber, C., Lühr, H., and Schwintzer, P.: First CHAMP Mission Results for Gravity, Magnetic and Atmospheric Studies, Springer, Berlin-Heidelberg, 2003.
Reigber, C., Lühr, H., Schwintzer, P., and Wickert, J.: Earth Observation with CHAMP, Results from Three Years in Space, Springer, Berlin-Heidelberg, 2005.

Yin, F. and Lühr, H.: Recalibration of the CHAMP satellite magnetic field measurements, Meas. Sci. Technol., 22, 055101, doi:10.1088/0957-0233/22/5/055101, 2011. 